\title{
Partial Morphologic Response
}

National Cancer Institute

\section{Source}

National Cancer Institute. Partial Morphologic Response. NCI Thesaurus. Code C123602.

Mixed attenuation and some homogeneity of the metastases with a partially resolved peripheral rim. 